\title{
Structures of Prosapogenin-B and $-A$ of Dioscin and Cooccurrence of B with Dioscin in the Rhizoma of Dioscorea Tokoro MAkivo')
}

\author{
Toshio Kawasaki and Tatsuo Yamauchi \\ Faculty of Pharmacentical Sciences, Kyushu University ${ }^{2)}$
}

(Received September 12, 1967)

\begin{abstract}
Prosapogenins, B and A, which are provided together with prosapogenin-C (diosgenin $\beta$-D-glucopyranoside) (II) on partial hydrolysis of dioscin (diosgenin 2-O- and 4-O-bis$\alpha$-L-rhamnopyranosyl- $\beta$-D-glucopyranoside $=$ diosgenin $\beta$-chacotrioside) (I) were characterized as 4-O- (III) and 2-O- $\alpha-\mathrm{L}-$ rhamnopyranosyl- $\beta-\mathrm{D}-$ glucopyranoside (IV), respectively.

A glycoside found along with dioscin, gracillin and other spirostanol glycosides in the rhizoma of Dioscovea Tokoro Makino was isolated and identified with prosapogenin-B of dioscin on the basis of paper, thin-layer and, for the first time, gas liquid chromatographic comparisons and chemical evidence.

The difference of the site of the probable enzymatic cleavage in the sugar portion (chacotriose) of dioscin from that of the splitting by acid bydrolysis was noted.
\end{abstract}

Dioscin, a steroid saponin (spirostanol glycoside) in the rhizoma of some Dioscorea plants, has been assigned the structure, diosgenin $2-\mathrm{O}-$ and $4-\mathrm{O}-$-bis- $\alpha-\mathrm{I}-\mathrm{rhamnopyranosyl-} \beta-$ D-glucopyranoside (diosgenin $\beta$-chacotrioside) (I). ${ }^{3)} \quad$ On its partial hydrolysis three prosapogenins are provided and have been named, in order of increasing polarity, prosapogenin-C, $-\mathrm{B}$ and $-\mathrm{A}$ of dioscin. $\mathrm{C}$ and $\mathrm{A}$ were isolated in a pure state and the former was identified as diosgenin $\beta-\mathrm{D}-$ glucopyranoside (trillin) (II), but $\mathrm{A}$ has only been known to be a $a-\mathrm{L}-$-rhamnopyranosyl- $\beta$-D-glucopyranoside and B which must be another $\alpha-\mathrm{L}-\mathrm{rhamnopyranosyl- \beta - \textrm {D } -}$ glucopyranoside has not been isolated because of its poor yield. ${ }^{3)}$ Thus the site of linkage of rhamnose to glucose, 2-O- or 4-O-, in the sguar moieties of $\mathrm{B}$ and $\mathrm{A}$ has remained unknown.

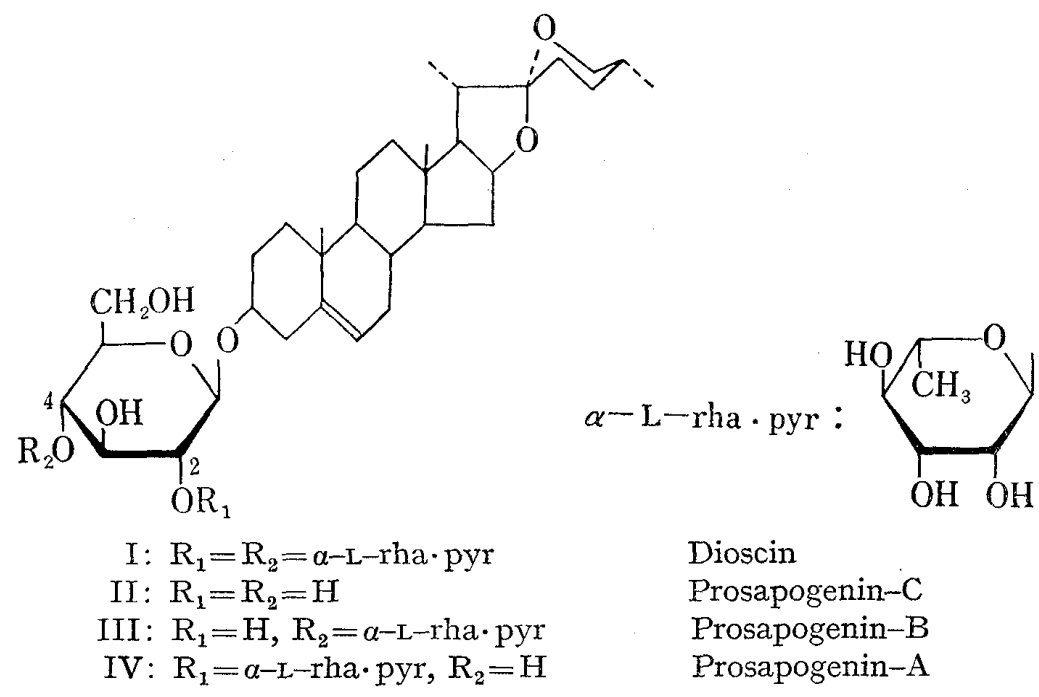

1) Presented at the Annual Meeting of the Pharmaceutical Society of Japan, in Sendai, Oct. 22, 1966.

2) Location: Katakasu, Fukuoka.

3) a) T. Tsukamoto, T. Kawasaki, and T. Yamauchi, Chem. Pharm. Bull. (Tokyo), 4, 35 (1956); b) T. Kawasaki and T. Yamauchi, ibid., 10, 703 (1962). 
In the meantime the water-insoluble fraction of the ethanol extract of the rhizoma of Dioscorea Tokoro was found independently by M. Goto, et al. ${ }^{4)}$ and in our laboratory to contain, along with dioscin, gracillin, and other spirostanol glycosides reported previously, ${ }^{5}$ ) a glycoside which was expected to be one of the prosapogenins of dioscin or gracillin.

This paper deals with the isolation using an improved method and characterization of prosapogenin-B and $-\mathrm{A}$ in a partial hydrolyzate of dioscin, and with the identification of the glycoside newly found in $D$. Tokoro as prosapogenin-B of dioscin.

As described earlier ${ }^{3 a}$ ) the isolation of small amount of prosapogeinin-B in the waterinsoluble product of a partial hydrolysis directly by means of chromatography on alumina was not successful, but acetylation of the product followed by chromatography on silica gel was found to give a good resolution of the components to afford B acetate as well as A acetate. The subsequent saponification regenerated the free glycosides which were homogeneous and corresponded to $\mathrm{B}$ and $\mathrm{A}$ on paper ${ }^{3 a}$ ) and thin-layer of silica gel. ${ }^{6}$

Prosapogenin- $\mathrm{B}$ thus obtained was a hygroscopic white powder, $\mathrm{mp} 215-220^{\circ}$ (decomp.), $[a]_{D}-96^{\circ}$ (pyridine), $\mathrm{C}_{39} \mathrm{H}_{62} \mathrm{O}_{12} \cdot 3 \mathrm{H}_{2} \mathrm{O}$ and gave on acid hydrolysis diosgenin, D-glucose and L-rhamnose. Complete methylation followed by methanolysis afforded a mixture of methyl glycosides of methylated sugars, which was found by gas liquid chromatography ${ }^{7}$ to consist of methyl 2,3,4-tri-O-methyl-L-rhamnopyranoside and methyl 2,3,6-tri-O-methylD-glucopyranoside. Therefore prosapogenin-B of dioscin is defined as diosgenin 4-O- $\alpha-$ L-rhamnopyranosyl- $\beta-\mathrm{D}-$ glucopyranoside (III). The structure of prosapogenin-A which should be $2-\mathrm{O}-\alpha$-L-rhamnopyranosyl- $\beta$-D-glucopyranoside (IV) was evidenced as such by identification of the methanolyzate of the permethylate as a mixture of methyl 2,3,4-tri-Omethyl-L-rhamnopyranoside and methyl 3,4,6-tri-O-methyl-D-glucopyranoside.

A glycoside (compound $\mathrm{x}$ ) which was found in the water-insoluble fraction of the ethanolic extract of the rhizoma showed a $R f$ value quite similar to that of prosapogenin-B of dioscin on thin-layer chromatogram. The isolation of compound $\mathrm{x}$ from a mixture of spirostanol glycosides was achieved by the conventional method followed by the afore-mentioned procedures, acetylation, silica gel chromatography, and saponification, to give a hygroscopic powder, $\mathrm{mp} 195-205^{\circ}$ (decomp.), $[\alpha]_{\mathrm{D}}-92^{\circ}$ (pyridine). It revealed a single spot corresponding to that of $\mathrm{B}$ on paper and thin-layer of silica gel, but the melting point could not be raised up to that of the authentic sample obtained by partial hydrolysis of dioscin. Therefore as a possible aid for further examination of purity and identity, the application of gas liquid chromatography was attempted.

There had been no record, $\left.{ }^{8}\right)$ to the authors' knowledge, of the previous gas liquid chromatography of steroid glycosides, but in view of the successful result of Furuya ${ }^{9}$ ) on some plant glycosides, eleven spirostanol glycosides were converted to permethyl ethers and pertrimethylsilyl ethers and subjected to chromatography. As shown in Table II, these derivatives gave the respective single peak seemingly without decomposition on silicon polymer $\mathrm{SE}-30$ even at about $300^{\circ}$ and the resolution was fairly satisfactory except for a few cases, for example dioscin and gracillin (triglycosides). Compound $\mathrm{x}$ permethylate was then chromatographed alone and on admixture with $B$ permethylate and in both cases a single peak

4) Private communication from Dr. M. Goto and Mr. S. Imai of the Research Laboratories of Takeda Chemical Industries, Itd.

5) T. Tsukamoto, T. Kawasaki, and T. Yamauchi, Yakugaku Zasshi, 77, 1225 (1957).

6) T. Kawasaki and K. Miyahara, Chem. Pharm. Bull. (Tokyo), 11, 1546 (1963).

7) G.O. Aspinall, J. Chem. Soc., 1963, 1676.

8) Quite recently gas liquid chromatographies of cardiac glycosides and related compounds ${ }^{10}$ ) and of spirostanol glycosides (Convallaria saponins) ${ }^{11}$ ) were reported.

9) T. Furuya, J. Chromatog., 18, 152 (1965).

10) W.E. Wilson, S.A. Johnson, W.H. Perkins, and J.E. Ripley, Anal. Chem., 39, 40 (1967).

11) M. Kimura, S. Tohma, I. Yoshizawa, and F. Fujino, Annual Meeting of the Pharmaceutical Society of Japan, in Kyoto, April 10, 1967. 
was observed. Therefore compound $\mathrm{x}$ was regarded to be homogeneous and identical with prosapogenin- $\mathrm{B}$ of dioscin. The identity was confirmed by the analytical data corrresponding to $\mathrm{C}_{39} \mathrm{H}_{62} \mathrm{O}_{12} \cdot 3 \mathrm{H}_{2} \mathrm{O}$, acid hydrolysis affording diosgenin, D-glucose and L-rhamnose, and unequivocally by characterization of methylated monosaccharides yielded on methanolysis of the permethylate.

In the course of isolation of compound $\mathrm{x}$ another substance which seemed most likely to be prosapogenin-A of dioscin was also obtained, but the yield was very low and it is apparent that the predominant one of the two isomeric $\alpha-L-$-rhamnopyranosyl- $\beta$-D-glucopyranosides is prosapogenin-B.

It is known that saponins and glycosidal steroid alkaloids (basic steroid saponins) having an oligosaccharide as their glycidic parts are often accompanied in nature by their corresponding prosapogenins which might be formed by an enzymatic degradation of the oligosaccharide portion of the glycosides. Among solanum alkaloids (glycosides of solanidane and spirosolane series) $\alpha$-chaconine, $\alpha$-solamargine and $\beta$-solamarine have the same sugar moeity, chacotriose, as that of dioscin, and they have been shown to be accompanied in the original plant, respectively, by $\beta$-chanonine, ${ }^{12,13)} \beta$-solamargine, ${ }^{13)}$ and $\gamma$-solamarine. ${ }^{14)}$ The former two have been regarded to be $4-\mathrm{O}-\alpha-\mathrm{L}-$-rhamnopyranosyl $-\beta-\mathrm{D}-$ glucopyranoside and the last one was proved to be as such.

Cooccurrence mainly of prosapogenin-B with dioscin is in good agreement with the cases of analogous basic saponins. The fact that the site of probable enzymatic cleavage in the chacotriose residue of dioscin is different from that of splitting by acid hydrolysis which yields 2-O-rhamnosyl glucoside as the major diglycoside is noteworthy. ${ }^{15}$ )

\section{Experimental ${ }^{18)}$}

Isolation of Prosapogenin-B and -A from the Partial Hydrolyzate of Dioscin-Dioscin (1.6 g) was hydrolyzed with $0.5 \mathrm{~N} \mathrm{HCl}$ in $50 \%$ EtOH $(160 \mathrm{ml})$ on refluxing for $1 \mathrm{hr}{ }^{3 a}$ ) EtOH was removed in vacuo, water added and the precipitates were extracted with $\mathrm{BuOH}$. BuOH layer was washed with water, evaporated in vacuo to dryness. The residue $(1.57 \mathrm{~g})$ was acetylated with $20 \mathrm{ml}$ each of pyridine and $\mathrm{Ac}_{2} \mathrm{O}$ at $100^{\circ}$ for $3 \mathrm{hr}$ to give a mixture of acetates $(1.92 \mathrm{~g})$. The product was placed on a column of silica gel (170 g) and eluted with benzene- $\mathrm{Me}_{2} \mathrm{CO} 10: 1$ and 5:1, successively: Fr. $1(10: 1,120 \mathrm{ml}) 1.06 \mathrm{~g}, \mathrm{Rf} 0.98,0.74$ (diosgenin acetate 0.98 , prosapogenin-C acetate 0.73$)$; Fr. $2(10: 1,30 \mathrm{ml}) 396 \mathrm{mg}, 0.73,0.54,0.51 ;$ Fr. 3 (5:1, $70 \mathrm{ml})$ $540 \mathrm{mg}, 0.51$. The above Fr. 2 was rechromatographed on silica gel (80 g) using benzene- $\mathrm{Me}_{2} \mathrm{CO} 12: 1$ as the solvent: Fr. 1' $(20 \mathrm{ml}) 33 \mathrm{mg}$, Rf 0.73; Fr. 2' $(20 \mathrm{ml}) 50 \mathrm{mg}, 0.54$; Fr. $3^{\prime}(20 \mathrm{ml}) 124 \mathrm{mg}, 0.54,0.51$; Fr. $4^{\prime}$ $(100 \mathrm{ml}) 130 \mathrm{mg}, 0.51$.

Prosapogenin-B__Fr. $2^{\prime}$ of the second chromatography was crystallized from $\mathrm{MeOH}$ to give a white cyrstalline powder $(32 \mathrm{mg}), \mathrm{mp} 115-120^{\circ},[\alpha]_{\mathrm{D}}-72^{\circ}\left(\mathrm{CHCl}_{3}\right)$. The acetate was boiled with $2 \% \mathrm{KOH}$ in

12) R. Kuhn, I. Löw, and H. Trischmann, Ber., 88, 1492, 1690 (1955).

13) R. Tschesche and G. Wulff, Planta Med., 12, 272 (1964).

14) P. M. Boll, Acta Chem. Scand., 16, 1819 (1962); ibid., 17, 1852 (1963).

15) The same difference is observed in chacotriose moiety of $\beta$-solamarine (cf. Fig. 1 and Table II of ref. 10)). Tschesche, et al.16) have reported that the mode of enzymatic breakage of sugar-sugar bond in the glycidic part of parillin was different from that of cleavage with acid. On the other hand, gracillin ( $\mathrm{rha}{ }_{\mathrm{glc}} \frac{1-2}{1-3}$ glc-diosgenin ) gave on acid hydrolysis no 2-O-rhamnosylglucoside but 3-O-glucosylglucoside, ${ }^{17}$ ) which was the predominant diglycoside coexistent with gracillin in the rhizoma of D. gracillima MIQ. (unpublished data).

16) R. Tschesche, R. Kottler, and G. Wulff, Ann., 699, 212 (1966).

17) T. Tsukamoto and T. Kawasaki, Chem. Pharm. Bull. (Tokyo), 4, 104 (1956); T. Kawasaki and T. Yamauchi, ibid., 10, 703 (1962).

18) All melting points were taken on Kofler block and are uncorrected. Unless otherwise stated $R f$ values were determined on thin-layer of silica gel $\mathrm{G}$ (Merck) using $\mathrm{CHCl}_{3}-\mathrm{MeOH}$-water 7:3:1 (for glycosides) and benzene-iso PrOH 20:1 (for glycoside acetates and methylates) as the solvents (cf. ref. 3). In column chromatography "Kanto" silica gel (100-200 mesh) was employed. Gas liquid chromatography wasrun with Yanagimoto Model GC-550F Gas Chromatograph equipped with a hydrogen flame ionization detector. 
$\mathrm{MeOH}(3 \mathrm{ml})$ for $45 \mathrm{~min}$ and the regenerated free glycoside was crystallized from $\mathrm{MeOH}$ to provide $\mathrm{B}$ as a hygroscopic white powder, mp $215-220^{\circ}$ (decomp.), $[\alpha]_{\mathrm{D}}-96^{\circ}$ (pyridine), Rf 0.46 (dioscin $0.28, \mathrm{~A} 0.38$, $\mathrm{B} 0.45, \mathrm{C} 0.5 \bar{i}$ ), $R f$ on paper chromatogram (benzene-BuOH-water $10: 4: 5^{3 a}$ ) 0.76 (dioscin 0.39 , A $0.61, \mathrm{~B}$ 0.75, C 0.91). Anal. Calcd. for $\mathrm{C}_{39} \mathrm{H}_{62} \mathrm{O}_{12} \cdot 3 \mathrm{H}_{2} \mathrm{O}$ (diosgenin rhamnosylglucoside trihydrate) : $\mathrm{C}, 60.21 ; \mathrm{H}, 8.81$. Found: $\mathrm{C}, 60.30 ; \mathrm{H}, 8.88$.

Prosapogenin- $\mathbf{A}^{3} a$ ___ Fr. 3 of the first chromatography was crystallized from $\mathrm{MeOH}$ to give colorless fine needles $(300 \mathrm{mg}), \mathrm{mp} 198-200^{\circ}$. The acetate $(280 \mathrm{mg})$ was saponified with $2 \% \mathrm{KOH}$ in $\mathrm{MeOH}(20 \mathrm{ml})$

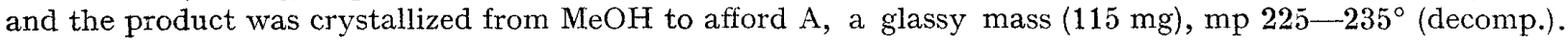
$R f$ values on thin-layer and paper run as in $B$ were 0.39 and 0.62 , respectively.

Methanolysis of Permethylates of Prosapogenin-B and -A, and Examination of the Resulting Methylated Sugars__ B and A were fully methylated by the Kuhn method ${ }^{3 b}$ ) and the respective permethylate (10 mg) $\left.{ }^{19}\right)$ which was homogeneous on thin-layer of silica ge ${ }^{20)}$ and showed no hydroxyl absorption in the IR spectrum was refluxed with $7 \% \mathrm{HCl}$ in $\mathrm{MeOH}(1 \mathrm{ml})$ for $3 \mathrm{hr}$. The reaction mixture was neutralized with $\mathrm{Ag}_{2} \mathrm{CO}_{3}$, the precipitates were filtered off and the filtrate was evaporated in vacuo. The residue was dissolved in a small amount of $\mathrm{Me}_{2} \mathrm{CO}$ and subjected to gas liquid chromatography. The result is shown in Table $\mathrm{I}$.

TABLE I. Gas Liquid Chromatography of Methylated Monosaccharides obtained by Methanolysis of Prosapogenin Permethylate

\begin{tabular}{|c|c|c|c|}
\hline \multirow{2}{*}{$\frac{\text { Methylated monosaccharides }}{\text { from Prosapogenin-B Permethylate }}$} & \multicolumn{3}{|c|}{ Relative retention time $\left.\mathrm{e}^{a}\right)$} \\
\hline & 1. 00 , & 8.20 , & 11.10 \\
\hline from Prosapogenin-A Permethylate & 1.00, & 7. 15, & 8.50 \\
\hline from Compound $\mathrm{x}$ Permethylate & 1.00, & 8.20 , & 11.10 \\
\hline Me 2,3,4-tri-O-Me-L-Rhamnoside & 1.00 & & \\
\hline $\mathrm{Me} 3,4,6$-tri-O-Me-D-Glucoside $\left.{ }^{b}\right)$ & & 7. 15, & 8.50 \\
\hline $\mathrm{Me} 2,3,6$-tri-O-Me-p-Glucoside $\left.{ }^{c}\right)$ & & 8.20 , & 11.10 \\
\hline $\left.\mathrm{Me} 2,4,6-\operatorname{tri}-\mathrm{O}-\mathrm{Me}-\mathrm{D}-\mathrm{Glucoside}{ }^{d}\right)$ & & 7.68, & 11.35 \\
\hline $\mathrm{Me} 2,3,4-$ tri--O-Me-D-Glucoside ${ }^{e)}$ & & 6.00 , & 8.65 \\
\hline
\end{tabular}

Conditions: Glass column $1.2 \mathrm{~m}$ long, $2 \mathrm{~mm} \phi$, packed with $5 \%$ 1,4-butanediol succinate ${ }^{7)}$ on Shimalite W $(60-80 \mathrm{mesh}) ; \mathrm{N}_{2}$ flow rate $28 \mathrm{ml} / \mathrm{min}$; flash heater temp. $210^{\circ}$; column temp. $138^{\circ}$; ; detector temp. $210^{\circ}$.

a) Relative to the retention time $(0.95 \mathrm{~min})$ of $\mathrm{Me} 2,3,4-\mathrm{tri}-\mathrm{O}-\mathrm{Me}-\mathrm{L}-\mathrm{rhamnoside}$

b) Prepared as reported by Sundberg, et al. (R.L. Sundberg, C.M. McCloskey, D.E. Rees, and G.H. Coleman, J. Am. Chem. Soc., 67, 1080 (1945).

c) Prepared on hydrolysis of lactose permethylate followed by glycosidation with $\mathrm{MeOH}-\mathrm{HCl}$.

d) Prepared by permethylation of prosapogenin $\mathrm{A}^{17}$ ) of gracillin followed by methanolysis.

e) Prepared according to Haworth, et al. (W.N. Haworth, E.L. Hirst, E.J. Miller, and A. Learner, J. Chem. Soc., 1927, 2443).

Isolation of Compound $\mathbf{x}$ in the Rhizoma of Dioscorea Tokoro MAKINo-Water-insoluble fraction of the EtOH extract of the rhizoma of $D$. Tokoro ${ }^{21)}$ was dissolved in $\mathrm{BuOH}$ saturated with water and the solution was treated with $2 \% \mathrm{NaOH}$ in water several times. Dark brown water layer, containing some more polar saponins, was removed and $\mathrm{BuOH}$ layer was washed with water and evaporated in vacuo. The residue was extracted with ether in order to remove less polar glycosides $\left(e . g\right.$. yononin, tokoronin $\left.{ }^{22}\right)$ ) and the insoluble portion was crystallized from $\mathrm{CH}_{2} \mathrm{Cl}_{2}-\mathrm{MeOH}$ 1:1. A crystalline powder separated out (consisting of mainly dioscin, gracillin and tokoronin) was filtered off and the mother liquor was evaporated in vacuo to dryness. The residue $(5 \mathrm{~g})$ was heated with pyridine $(30 \mathrm{ml})$ and $\mathrm{Ac}_{2} \mathrm{O}(50 \mathrm{ml})$ at $100^{\circ}$ for $\left.3 \mathrm{hr}^{23}\right)$ and the crude acetate $(4.2 \mathrm{~g})$ was passed through a column of silica gel $(210 \mathrm{~g})$ using benzene-Me $\mathrm{MeO}_{2} \mathrm{10:1}$ as an eluant: Fr. $1814 \mathrm{mg}$, Rf 0.56 ; Fr. 22.0 g, 0.47; Fr. $3127 \mathrm{mg}, 0.46,0.38$; Fr. $4335 \mathrm{mg}, 0.39$; Fr. $5560 \mathrm{mg}, 0.1-0.2$ (Rf values of reference compounds: dioscin peracetate ${ }^{23)} 0.47$, gracillin acetate $\left.{ }^{17}\right) 0.39, \mathrm{~B}$ acetate $0.54, \mathrm{~A}$ acetate 0.51 , kikubasaponin acetate ${ }^{24}$ 0.15). A free glycoside obtained by alkali hydrolysis of Fr. 1 was found to be

19) Permethylate of $\mathrm{A}$ was crystallized from $\mathrm{MeOH}$ to give colorless needles, $\mathrm{mp} 154-156^{\circ},[\alpha]_{\mathrm{D}}-94^{\circ}$ $(\mathrm{MeOH})$.

20) $R f$ values of permethylates of $\mathrm{B}, \mathrm{A}$ and reference compounds were as follows: $\mathrm{B} 0.17, \mathrm{~A} 0.16$, dioscin 0.09 , gracillin 0.11 .

21) Cultivated in Kyoto and harvested in November.

22) T. Kawasaki and T. Yamauchi, Yakugaku Zasshi, 83, 757 (1963).

23) To be published (presented at the Annual Meeting of the Pharmaceutical Society of Japan, in Sendai, Oct. 22, 1966).

24) T. Kawasaki, T. Yamauchi, and R. Yamauchi, Chem. Pharm. Bull. (Tokyo), 10, 698 (1962). 
compound $\mathrm{x}$ contaminated with tokoronin and placed again on a column of silica gel (70 g) and eluted with $\mathrm{CHCl}_{3}-\mathrm{MeOH}$-water 7:3:1: Fr. $1475 \mathrm{mg}$, Rf 0.45 (B 0.46), 0.48 (tokoronin 0.48); Fr. $2350 \mathrm{mg}, 0.45 ; \mathrm{Fr} .3$ trace; Fr. $410 \mathrm{mg}, 0.38$ (A 0.39 ).

Compound $\mathrm{x}$-Fr. 2 in above chromatography was crystallized from MeOH to give compound $\mathrm{x}$ as a hygroscopic white powder, mp $195-205^{\circ}$ (decomp.), $[\alpha]_{\mathrm{D}}-92^{\circ}$ (pyridine). Anal. Calcd. for $\mathrm{C}_{39} \mathrm{H}_{62} \mathrm{O}_{12}$. $3 \mathrm{H}_{2} \mathrm{O}$ (diosgenin rhamnosylglucoside trihydrate) : $\mathrm{C}, 60.21 ; \mathrm{H}, 8.81$. Found: $\mathrm{C}, 60.02 ; \mathrm{H}, 8.88$. Thin-layer and paper chromatographies run in parallel, and on admixture, with II showed the homogeneity and the identity. Ten $\mathrm{mg}$ was hydrolyzed with $2 \mathrm{~N} \mathrm{HCl} \mathrm{in} 50 \%$ EtOH $(2 \mathrm{ml})$ on refluxing for $2 \mathrm{hr}$, treated as described before $^{3 a}$ ) and the product was examined by thin-layer ${ }^{25}$ (for aglycone) and paper (for sugars) ${ }^{3 a}$ ) chromatographies. Diosgenin, D-glucose and L-rhamnose were detected. Permethylation, methanolysis, and examination of the methylated sugars yielded were conducted in the same way as in B and the result is shown in Table $\mathrm{I}$.

Gas Liquid Chromatography of Spirostanol Glycosides

Materials: All glycosides except for those described below had been isolated or prepared in this laboratory.

Prosapogenin- $\mathrm{B}\left(\mathrm{BDH}_{2}\right)$ and $-\mathrm{A}\left(\mathrm{ADH}_{2}\right)$ of Dihydrodioscin: Dihydrodioscin, $\left.{ }^{26}\right) \mathrm{mp} 292-295^{\circ}$ (decomp.) (from dil. EtOH), $[a]_{D}-91^{\circ}$ (pyridine), prepared by hydrogenation of dioscin peracetate ${ }^{23}$ ) over $2 \% \mathrm{Pd}-\mathrm{C}$ in $\mathrm{MeOH}$ followed by alkali hydrolysis was refluxed with $1 \mathrm{~N} \mathrm{H}_{2} \mathrm{SO}_{4}$ in $50 \% \mathrm{EtOH}$ for $0.5 \mathrm{hr}$. The waterinsoluble product $(R f 0.97,0.66,0.55,0.49,0.38$, dihydrodioscin 0.38 ) was fractionated by repeated column chromatographies first on alumina (Woelm, grade 4) (solvent: $\mathrm{CHCl}_{3}-\mathrm{MeOH} 10: 1,5: 1$ and $\mathrm{BuOH}$ saturated with water) and then silica gel (solvent: $\mathrm{CHCl}_{3}-\mathrm{MeOH}$-water $7: 3: 1$, AcOEt-EtOH-water $3: 1: 3$ or 2:1:2). $\mathrm{BDH}_{2}$ and $\mathrm{ADH}_{2}$ fractions (checked on thin-layer) were crystallized from $\mathrm{MeOH}$, respectively, to give a white powder, $\operatorname{mp} 165-186^{\circ}$ (decomp.), Rf 0.55 and a crystalline powder, $\mathrm{mp} 254-259^{\circ}\left(\right.$ decomp.), $[\alpha]_{\mathrm{D}}-84^{\circ}$ (dioxane), Rf 0.49 . Both gave on acid hydrolysis tigogenin (by thin-layer chromatography $y^{25)}$ ), D-glucose and L-rhamnose (by paper chromatography $\left.{ }^{3} a\right)$ ).

Methylation: Carried out according to the Kuhn method ${ }^{36)}$ and Hakomori method. ${ }^{27)}$

Trimethylsilylation: Carried out principally in the same manner as described by Furuya.9) The reaction mixture was evaporated in vacuo and the trimethylsilyl ether was dissolved in benzene for injection.

Conditions and results: Shown in Table II.

TABLE II. Gas Liquid Chromatography of Spirostanol Glycosides

\begin{tabular}{|c|c|c|c|c|c|}
\hline \multirow{3}{*}{ Glycoside } & \multirow{3}{*}{ Sugar Moiety } & \multicolumn{4}{|c|}{ Relative retention time ${ }^{a)}$} \\
\hline & & \multicolumn{2}{|c|}{ Methyl ether } & \multicolumn{2}{|c|}{ Trimethylsilyl ether } \\
\hline & & $1^{b)}$ & $2^{c)}$ & $1^{b)}$ & $2^{c)}$ \\
\hline Yononin $^{22)}$ & Ara & & & 0.4 & \\
\hline Tokoronin 22$)$ & Ara & & & 0.5 & \\
\hline Prosapogenin-C of Dioscin ${ }^{3)}$ & Glc & 1.0 & 1.0 & 1.0 & 1.0 \\
\hline Prosapogenin-B of Dioscin & Rha + Glc & 4.7 & 4.6 & 2.7 & \\
\hline Prosapogenin-A of Dioscin & Rha + GIc & 3.6 & 3.5 & 2.5 & \\
\hline Prosapogenin-A of Gracillin ${ }^{17}$ ) & $\mathrm{Glc}+\mathrm{Glc}$ & & & 4.6 & 4.2 \\
\hline Prosapogenin-B of Dihydrodisocin & Rha + Glc & 4.6 & 4.5 & 2.6 & 3.0 \\
\hline Prosapogenin-A of Dihydrodioscin & $\mathrm{Rha}+\mathrm{Glc}$ & 3.8 & 3.8 & & \\
\hline Timosaponin $\mathrm{A}-\mathrm{II}^{d}$ ) & $\mathrm{Glc}+\mathrm{Gal}$ & 3.5 & & & \\
\hline $\left.\operatorname{Dioscin}^{3}\right)$ & $2 \mathrm{Rha}+\mathrm{Glc}$ & 13.0 & 9.5 & 7.5 & 8.2 \\
\hline Gracillin $^{17)}$ & Rha +2 Glc & 12.3 & & 8.8 & 8.0 \\
\hline Compound $\mathrm{x}$ & & 4.5 & & & \\
\hline
\end{tabular}

a) Relative to the retention time of the ether of prosapogenin-C of dioscin: methly ether; $0.68 \mathrm{~min}$ in condition $1,0.35 \mathrm{~min}$ in condition 2 . trimethylsilyl ether; $1.10 \mathrm{~min}$ in condition $1,0.50 \mathrm{~min}$ in condition 2.

b) Glass column, $120 \mathrm{~cm}$ long, $2 \mathrm{~mm} \phi$, packed with $1.5 \% \mathrm{SE}-30$ on Chromosolv $\mathrm{W}(60-80 \mathrm{mesh}) ; \mathrm{N}_{2}$ flow rate $58 \mathrm{ml} / \mathrm{min}$; flash heater temp. $355^{\circ}$; column temp. $290^{\circ}$; detector temp. $330^{\circ}$.

c) Glass column, $60 \mathrm{~cm}$ long, $3 \mathrm{~mm} \phi$, packed as $(b) ; \mathrm{N}_{2}$ flow rate $154 \mathrm{ml} / \mathrm{min}$; flash heater temp. $310^{\circ}$; detector temp. $330^{\circ}$; column temp. $288^{\circ}$.

d) T. Kawasaki, T. Yamauchi, and N. Itakura, Yakugaku Zasshi, 83, 892 (1963); T. Kawasaki and T.!Yamauchi, Chem. Pharm. Bull. (Tokyo), 11, 1221 (1963).

25) N. Matsumoto, Chem. Pharm. Bull. (Tokyo), 11, 1189 (1963).

26) Dihydrogracillin prepared in the same way shows $\mathrm{mp} 276-279^{\circ}$ (decomp.), $[\alpha]_{D}-74^{\circ}$ (pyridine).

27) S. Hakomori, J. Biochem. (Japan), 55, 205 (1964). 
Acknowledgement The authors are grateful to Dr. M. Goto and Mr. S. Imai of the Research Laboratories of Takeda Chemical Industries, Ltd. for the extract of the rhizoma of $D$. Tokoro and for optical rotations of some samples. They are also indebted to Mr. K. Miyahara for checking the gas liquid chromatography of spirostanol glycosides, to Miss K. Sakai for technical assistance and to the members of the Central Analysis Room of this University for microanlaytical data. The work was supported in part by a Grant-in-Aid of Scientific Research from the Ministry of Education of Japan, to which the authors' thanks are due. 